\title{
Los procesos internos y la globalización
}

\author{
Carlos Mateo Balmelli
}

Este ensayo trata de desmerecer el enfoque del economicismo reduccionista que considera en exclusividad el factor económico de la globalización en detrimento de otros aspectos. En este sentido, aquí se considera la globalización como un proceso que responde a una heterogeneidad de elementos y que su vigencia se constata por la mayor incidencia que lo externo asume sobre los asuntos domésticos. Las conclusiones a las que se arriba tienden a plantear la reconceptualización del modelo de Estado-nación fundado y organizado a partir del sacrosanto principio de la soberanía absoluta. Además, se sugiere impulsar a nivel nacional los procesos de ordenamiento social que tengan como objetivo elevar la capacidad endógena de los países y ampliar el reconocimiento de los derechos de la ciudadanía política y social.

1.- El aumento de la capacidad endógena como respuesta a la complejidad del entorno internacional.

El final del siglo se acerca a pasos agigantados coincidiendo su finitud cronológica con transformaciones que afectan a la sociedad internacional y a las sociedades nacionales.

El final de la bipolaridad ideológica y militar, la globalización de las comunicaciones y las finanzas, los reclamos de reconocimiento y ampliación de los derechos de ciudadanía, los problemas medioambientales, la delincuencia, el reconocimiento universal de la promoción de los derechos humanos, están señalando la necesidad de cambios en los enfoques analíticos a través de los cuales se diseñan las políticas públicas y de los mecanismos institucionales con los que se deciden y llevan a cabo las tareas gubernamentales.

La modernidad como proceso social controvertido requiere que se establezcan estructuras institucionales que impulsen y sean capaces de conducir y conectar los procesos externos con los internos de moderni- 
zación. La emergencia de conflictos y problemas que cobran dimensión planetaria y la sobreabundancia de exigencias y situaciones obligan a rechazar los paradigmas explicativos que establecían una relación unívoca entre un factor y el desarrollo.

Actualmente, en las ciencias sociales el desarrollo como posibilidad histórica es pensado a través de modelos analíticos que ponderan un conjunto de variables. La probabilidad de concretizar la sociedad desa $\neg$ rrollada en la que lo posible sea más que lo necesario, aumenta con el uso racional de los recursos y con la disponibilidad coincidente de los factores políticos, institucionales, económicos y sociales.

El elevado nivel de complejidad y el exceso de restricciones exigen establecer instituciones capaces de constituirse en ordenadores de los nuevos procesos y circunstancias que se suceden a nivel local, nacional e internacional:-

Crear instituciones y fortalecer las existentes es la respuesta política a lás demandas del cambio social. La solución de los problemas sociales está en el ámbito de la política. Descle la matriz política se debe concretizar el entorno institucional. El momento actual del proceso histórico universal obliga a pensar lo impensable, pero respetando los hipervalores de la democracia. ${ }^{\text {I }}$

Las demandas provenientes del proyecto de modernidad imponen cambios en la agenda de gobierno y en la capacidad instalada de los Estados. Las reformas requeridas abarcan áreas diferentes de las instituciones públicas.

Cuando la vocación de reforma se confronta a la complejidad de la realidad nacional e internacional, la dirigencia debe implementar una estrategia de desarrollo institucional que suponga la toma de decisiones adecuadas para mejorar el estilo de gerenciamiento público y se ponga en marcha un proceso decisional y ejecutivo que racionalice el uso de los recursos disponibles.

La dirigencia política y social que directa o indirectamente influye en el proceso de formación de la voluntad política del Estado, para poder orientar el proceso político, debe pensar en términos históricos y no reducir su visión a lo inmediato. La complejidad social resultado del aumento de las interacciones obliga a estrechar los lazos entre conocimiento y decisión.

El conocimiento debe estar incorporado al proceso decisional, ésta es una de las formas a través de la cual los diseñadores de las políticas

\footnotetext{
'Yehezkel Dror, La capacidad de gobernar. Informe al Club de Roma, (Barcelona: 1994), p. 13.
} 
públicas podrán anticipar las necesidades del proceso social. Los actores políticos y sociales deben estar preocupados en administrar el proceso social y no los detalles de él.

Las élites gobernantes deben definir nuevos patrones de gobierno. ${ }^{2}$ La gobernabilidad en todos los niveles debe estar garantizada y debe comprender tareas que apuntalen el proceso de creación institucional y que introduzcan eficiencia y eficacia en la gestión gubernamental. El buen gobierno es aquel capaz de forzar y conducir los cambios sociales necesarios sin que por ello, al satisfacer las demandas del presente, se esté comprometiendo la capacidad de dar respuestas de las futuras generaciones a sus propias necesidades.

El objetivo de este ensayo es reflexionar y describir las transformaciones a las cuales están sometidas las unidades nacionales. Para emprender esta tarea analítica se parte del supuesto de la correlación existente entre los procesos internos y externos que se suceden entre las unidades nacionales y la sociedad internacional.

Gráficamente se puede dar a entender estas circunstancias como que la relación entre los términos es circular. Partiendo de este supuesto axiomático se puede llegar a comprender las dificultades a las cuales se enfrentan los gobiernos, y se puede comprender por qué resulta imperativa la labor de que los gobiernos disminuyan sus vicios y aumenten sus virtudes.

Los gobiernos están comprometidos a elevar sus capacidades administrativas. El aumento de la complejidad disminuye las probabilidades del buen gobierno, por eso se debe comprender que la calidad del proceso gubernamental es un factor determinante de las posibilidades de desarrollo.

Los gobiernos deben aumentar su capacidad de maniobra para direccionar las reformas necesarias hacia el mejoramiento de la capacidad endógena local. Para insertarse en el mundo global hay que ordenar la casa; esto significa elaborar una agenda que contenga capítulos relacionados con el fortalecimiento institucional, la estabilidad económica, la reforma financiera, el estímulo de los gobiernos locales, la descentralización administrativa del gobierno central, etc.

El objeto principal de la reforma es el Estado, que para los fines explicativos de este ensayo será desglosado como objeto de estudio en diferentes aspectos. En un primer momento se llevará a cabo la descripción del nuevo perfil que va cobrando el Estado-nación en un mundo

${ }^{2}$ Ibid., p. 14. 
globalizado; para.tal efecto, se hará un diagnóstico situacional de la sociedad internacional post Segunda Guerra Mundial. Seguidamente, desde un enfoque prospectivo, se tratará de èntender y se recomendará cómo se deben proyectar los Estados nacionales en la comunidad internacional.

Será una constante en este ensayo la preocupación de que los países se esfuercen en someter sus procesos internos de ordenación a criterios racionales. La racionalización debe ser incorporada en los procesos de ordenamiento interno por ser un imperativo de la modernidad y porque además los procesos internos de ordenación se realizan coincidiendo con la globalización de los fenómenos sociales.

La agenda que se elabore para elevar la capacidad endógena de los Estados debe incluir un capítulo sobre la imperiosa necesidad de consolidar un sistema financiero fundado sobre los principios de solvencia y transparencia.

Las políticas de reforma financiera se dirigen a establecer un régimen institucional estructurado sobre bases y condicionamientos homogéneos. La crisis del endeudamiento externo ha demostrado la vulnerabilidad de las unidades económicas nacionales cuando en ellas la inversión local no está vinculada al anorro doméstico. Por eso, para aumentar la capacidad endógena es recomendable encauzar las políticas que conduzcan a lograr el fortalecimiento institucional y patrimonial de la industria bancaria y de otras fuentes alternativas de financiación.

La propuesta consiste en dotar al régimen financiero de los recursos que posibiliten establecer vínculos estrechos entre la capacidad de ahorro interna y la inversión. De más está decir que las politicas de reforma financiera deben ser implementadas en un ambiente de estabilidad macroeconómica. Las políticas de ajuste hàn demostrado la inviabilidad de un modelo de desarrollo sustentable que desconozca la necesidad de mantener la disciplina fiscal y monetaria.

La estabilidad macroeconómica es un requisito para fortalecer el sistema financiero local y sus posibilidades de ahorro e inversión, come tambien en una condición ineludible de los esfuerzos integracionistas que someten sus posibilidades de éxito al logro de la convergencia macroeconómica. De lo que se acaba de afirmar no se puede inferir que la estabilidad macroeconómica per se produce crecimiento económico y desarrollo social. Las políticas públicas que pretendan aumentar la capacidad interna de los países deben ser formuladas considerando el uso racional de los recursos existentes en un ámbito económico estable. 
En este ensayo se considera prioritaria la necesidad de emprender una reforma integral del Estado. La agenda para la concresión de ese fin debe reconocer la necesidad demodernización de la administración pública, como también la de ejecutar políticas que tiendan a la vigorización de los gobiernos locales. El emprendimiento de esta tarea debe realizarse persiguiendo dos objetivos, que a un nivel abstracto puede ser planteado en términos dicotómicos.

En teoría se puede establecer que el mejoramiento de la gestión pública contradice la ampliación de la participación política. Sin embargo, el desafío de las políticas de reforma consiste, justamente, en hacer coincidir en la práctica aquello que en abstracto puede ser presentado como dicotómico.

El enfoque a través del cual será realizado este trabajo parte del supuesto de que el desarrollo es un fenómeno multidimensional; de allí que se puede deducir la compatibilidad de alcanzar un mejoramiento de gestión, al mismo tiempo que se solidifiquen los mecanismos de participación y representación política.

La reforma del Estado debe apuntalar el sofisticamiento de los esquemas institucionales que hagan funcionar racionalmente a la estructura estatal. La propuesta consiste en aumentar la capacidad de gerenciamiento público y dotar de mayor representatividad al conjunto de instituciones que configuran la sociedad política.

\section{2.- El condicionamiento interno y externo.}

La pretensión de teorizar sobre, quizás, el tema más importante de la filosofía política, es una tarea cuyo resultado final nunca satisface.

La conceptualización del Estado fue siempre objeto de reflexión de la ciencia politica. En la actualidad el debate de la reforma del Estado es argumentado a partir de modelos explicativos nuevos. Se puede constatar que la práctica estatal internacional de los diferentes Estados ha tenido que cambiar sus enfoques, sus delineamientos, sus agendas y prioridades debido a la transformación del contexto internacional.

Es por eso que se quiere enunciar y presentar algunas reflexiones que van a estar presentes en el transcurso de toda la exposición. En primer lugar, el imperativo contextual externo obliga a que se redefina el Estado-nación, lo que no excluye que desde la perspectiva de este ensayo las razones de dentro, que resultan de un nuevo relacionamiento entre el Estado y la sociedad, sean tenidas en cuenta en el análisis. 
Desdè este punto de vista en el análisis de la reconceptualización del Estädo-nación deben considerarse los condicionamientos externo e interno. Para poder entender a cabalidad la metamorfosis a la cual está sòmetido el Estado es necesario recurrir a un modelo integrado en el que se conisideren variables endógenas y exógenas. ${ }^{3}$

El concepto Estado-nación resultante de la postguerra y de la Guerra Fría configura una institucionalidad jurídica ineaapaz de poder actuar y reaccionar ante los nuevos desafios de los procesos sóciales globali$\operatorname{zados}^{4} \mathrm{y}$ de las nuevas demandas sociales y expectativas de pârticipación políticas internas. ${ }^{5}$

Los nuevos desafíos internos y externos limitan la capacidad operativa del Estado-nación clásico. La sobreabundancia de relaciones, posibilidades y necesidades impone la transformación institucional del Estado como un imperativo del proceso de modernización para el cual es necesario establecer zonas de cooperación en donde puedan acoplarse y complementarse los mecanismos jurídicos normativos nacionales con los internacionales.

En segundo lugar, es importánte destacar que los procesos de redefinición del Estado que conducen a instancias supranacionales de integración, por más que tengan un fuerte contenido funcionalista 0 respondan a necesidades del proceso económico, son en todos los casos antecedidos por el proceso político.

Se debe destacar que por más de que se esté de acuerdo con la necesidad de la diplomacia para el desarrollo ${ }^{6}$ y de considerar prioritaria la necesjdad de que los países se preparen endógenamente para insertarse en la sociedad internacional, consideramos que todo el proceso está subordinado a la voluntad y a la decisión política que le antecede.

Esas son aclaraciones conceptuales de fundamental importancia porque los procesos de integración, los procesos de creación de sociedades políticas, que solamente tengan como fundamento el interés pragmáticoeconómico, aparte de ser frágiles son reversibles. Es por eso el acierțo de considerar la decisión y la voluntad política, y el anhelo de asociarse en un nuevo modelo de comunidad supranacional, como un presupuesto

\footnotetext{
${ }^{3}$ Carlos Mateo Balmelli, El desarrollo institucional, (Asunción: 1995), pp. 237-279.

${ }^{4}$ Luciano Tomassini, La reforma del Estado y las politicas públicas, (Santiago de Chile: 1994), p. 57

${ }^{5}$ Roberto Russell, Los cambios actuales en la politica exterior jel palacio San Martín: contextos diferentes y reducción del mapamundi", en: José Luis Simón (ed.), Política exterior y democracia en el Paraguay y sus vecinos, (Asunción: 1995), pp. 155-158.

${ }^{6}$ Manfred Wilhelmy, De Aylwin a Frei: Continuidad polftica e intemacional", en: José L. Simón (ed.), op. cii., p. 173.
} 
que no niegue el Estado-nación y que tenga como primer fundamento el poder local, fundado y organizado en el régimen jurídico del Estadonación. $\therefore$.

$\because$ Es dê destacar que la estructura base de las instancias supranacionales en proceso de creación, es el marco jurídico político del Estado-nación. De conformidad con esto se concibe al Estado-nación como la única instancia capaz de resolver los problemas provenientes de la ciudadanía. Esto último se debe a dos razones: si bien es cierto que los procesos se globalizan, la gestión se localiza y en otro orden de cosas no se debe olvidar que el concepto de ciudadanía se compone de un elemento de lealtad política y por las circunstancias presentes, es correcto considerar inviable que se pueda producir una transferencia de lealtad política del Estado-nación a instancias supranacionales.

Siguiendo a Margaret Thatcher, ${ }^{7}$ se puede ameritar a favor de un internacionalismo construido sobre la base del Estado-nación. Todo proyecto internacionalista que pretenda suplantar al Estado-nación no alcanzará los objetivos que se propone. Cualquier institución supranacional carecerá de la representación política necesaria que le permita convocar los esfuerzos individuales y colectivos para la defensa y la promoción de las normas civilizadas de conducta internacional. Un proyecto internacionalista que niegue la función y la pertenencia del Estado-nación en la comunidad internacional está llamando a perecer y debilitar los elementos configuradores del concepto de ciudadanía.

La lealtad política hacia determinadas instituciones es un elemento definidor de la ciudadanía. Esta última, solamente puede ser concebida a partir del enfoque que incorpora la autonomía moral de las personas. En otro orden de cosas, el Estado-nación, a través de un proceso interno de vigorización del poder local, puede resolver los problemas provenientes de la demanda de derecho de ciudadanía social. Con la descentralización se amplía la participación y se mejora la capacidad de gestión de la administración pública por varias razones: una de ellas es que acerca la necesidad a la decisión.

Manfred Mols, ${ }^{8}$ en oposición a aquéllos que pronostican la paulatina desaparición del Estado-nación y refiriendo las circunstancias post Guerra Fría, sostiene que la estructura principal que describe a la sociedad internacional es el incremento de la interdependencia en ausencia de un modelo ordenador del proceso global.

\footnotetext{
${ }^{7}$ Margaret Thatcher, Los años de Downing Sireet, (Buenos Aires: 1993), p. 23.

${ }^{8}$ Manfred Mols, La sociedad del mañana, Cuadernos del CLAEH, No68, Montevideo, 1993, pp. 30-32.
} 
Siguiendo con el autor, se colige que ninguna de las instituciones de reciente creación y concurrentes con el Estado-nación posee una legitimación democrática propia; esto supone en el caso de esterilizar la capacidad decisional del Estado-nación se estará configurando una comunidad internacional administrativa e integrada por instancias tecnocráticas. También se debe agregar que lasorganizaciones competitivas del Estado-nación no poseen infraestructura administrativa propia, es decir: el Estado-nación con sobreabundancia o con precariedad infraestructural es la única instancia decisoria y ejecutiva con capacidad propia de actuar.

\section{3.- El desarrollo del Estado-nación.}

A continuación se harán algunas precisiones conceptuales y se caracterizará lo que fue en un inicio el Estado-nación moderno. En estas consideraciones no se tendrá como referencia inmediata al Estado latinoamericano.

El desarrollo del Estado-nación en Europa supuso la monopolización del ejercicio del poder político. Esto quiere decir, que, en el contexto europeo, la configuración de la instancia Estado-nación significó la expropiación del poder político de los centros feudales. En otros términos, se concentró, monopolizó y racionalizó el uso legítimo de la fuerza a través del Estado, considerando a las instancias públicas fundadas sobre el principio absoluto de soberanía. ${ }^{9}$

En Europa, el Estado moderno fue la respuesta a un proceso social de mayor complejidad. Razones que tienen que ver con las necesidades de una sociedad moderna y burguesa encuentran en la emergencia del Estado-nación la respuesta institucional válida para las demandas endógenas de modernización.

Desde esta perspectiva se puede entender la necesidad de la centralidad institucional del Estado como un factor condicionado por variables económicas que estaban vinculadas a las exigencias de concreción de un mercado único. Las exigencias de un mercado emergente requería la instauración de una instancia decisional integradora que consolidase un sistema jurídico uniforme. ${ }^{10}$

\footnotetext{
${ }^{9}$ Max Weber, Wirtschaft und Gesellschaft, (Tubingen: 1972), pp. 815-837.

${ }^{10}$ Hermann Heller, Staaslehre, (Tubingen: 1983), pp. 150-155.
} 
El proceso de configuración del:Estado-nación europeo condujo a la profesionalización y modernización de los ejércitos. ${ }^{11}$ Este proceso de cambio terminó con la transformaoión del ejército feudal en ejército nacional. Este último fue un elemento de considerable importancia para consolidar el Estado moderno. El ejército profesional y nacional sirvió de garantía y reaseguro del poder central y tuvo el efecto de afirmar el principio de la soberanía absoluta.

Otro elemento importante en el procéso de institucionalización del Estado moderno: fue la creación:en el ámbito nacional de un sistema jurídico uniforme. Esto se expresóien la concreción del Estado como unidad jurídica, es decir: lài universalización en un espacio geográfico determinado, de un sistema jurídico.

Estas características que se hanenunciado deben ser tenidas en cuenta cuando se está pretendiendo anticipar el nuevo perfil que va cobrando el Estado. Como se dijo, el Estado-nación sufre una metamorfosis, que es consecuencia de las circunstancias cambiantes de la sociedad internacional y de las atinentes a las demandas internas.

El modelo de Estado-nación clásico condujo a consolidar un sistema internacional en el cual la soberanía absoluta implicaba el exclusivismo jurídico del derecho nacional. ${ }^{12}$ El Estado era la única instancia creadora del derecho y en lo político la cooperación estaba pensada en términos de alianzas político-militares. Estos elementos condicionaron la emer:gencia de un sistema internacional competitivo y anarquizado en el cual se encontraban más áreas en conflictos y en competencias que en cooperación entre los diferentes actores.

\section{4.- La transformación de las relaciones internacionales.}

En el pasado la conducta de los Estados estaba de sobremanera influenciada por la desconsideración y la desconfianza hacia el otro. En la actualidad la

interdependencia en ciernes sugiere que el empobrecimiento del vecino no es la mejor fórmula para satisfacer las demandas nacionales de la ciudadanía. ${ }^{13}$

\footnotetext{
${ }^{11}$ paul Kennedy, The Rise and Fall of the Great Powers, (Estados Unidos: 1989), p. 75.

${ }^{12}$ Hans Kelsen, Principio del derecho internacional puiblico, (Buenos Aires: 1965), p. 381.

${ }^{13}$ Robert Keohane/Joseph Nye, Poder e interdependencia. La politica mundial en transición, (Argentina: 1988).
} 
En el presente se corrobora que la política exterior de los Estados se diseña y ejecuta a partir de consideraciones de diversa índole.

Son otras las variables que se consideran en las diferentes cancillerías, no solamente de la región sino en todo el mundo. El primer supuesto o la primera condición que empieza a ser modificada o revertida es el enfoque de lo que Kelsen, ${ }^{14}$ con muy buen criterio, llamaba, desde un punto de vista jurídico, el solipsismo, es decir: el subjetivismo radical, el reconocer solamente el propio yo.

El solipsismo jurídico es hoy en día una posición doctrinaria insuficiente para la planificación de la construcción de un nuevo orden internacional. Se impone hoy en día en toda política internacional, si es que se va a hablar de la construcción de órdenes institucionales supranacionales, que los Estados reconozcan la necesidad de la alteridad; vale decir reconocer la necesidad del otro para cooperar en política internacional. Tratar de superar el esquema atomizado de ver a los Estados como comunidades átomos y entender la necesidad de lograr una acción sistémica encaminada a solucionar problemas comunes.

Se debe pensar en zonas de cooperación como un elemento integrador, que convierta a las políticas exteriores en instrumento para el desarrollo social y favorezca la consolidación de la democracia. ${ }^{15}$

El subjetivismo radical en lo jurídico y político fue lo que caracterizó al Estado-nación previo a la Segunda Guerra Mundial. Desde el final de la última guerra mundial el Estado ha sufrido y está sufriendo una transformación acelerada, especialmente porque el mundo se ha vuelto interdependiente e integrado geográficamente. Los medios de comunicación, las demandas internas, las razones de dentro, están obligando a que el entorno internacional vaya cambiando y se vayan creando mecanismos de integración y de decisión a nivel internacional, que puedan, de alguna manera, establecer marcos de cooperación.

Paul Kennedy en su último libro Preparing for the Twenty-First Century (1993) corrobora la metamorfosis a la que está sometido el Estado en la actualidad. Para este autor la transformación del Estado se expresa en la relocalización de la autoridad dentro y fuera de la geografía nacional.

Es constatable en todas las sociedades nacionales que el Estado en su versión tradicional está siendo redefinido. Se da un proceso paradójico que puede concluir con resultados ambiguos; es decir: con asimetrías

\footnotetext{
${ }^{14}$ Hans Kelsen, op. cit.

${ }^{15}$ Manfred Wilhelmy, op. cil.; Roberto Russell, op. cit.
} 
institucionales, pues el Estado nacional se descentraliza para adentro y transfiere capacidad decisional hacia afuera. Las asimetrías institucionales deben ser reducidas a su mínima expresión pues ellas se convierten en un obstáculo en el proceso de elaboración de mecanismos jurídicos que posibiliten el acoplamiento de los sistemas jurídicos nacionales a los sistemas supranacionales.

$s$

El establecimiento de vasos vinculantes entre la sociedad nacional y la sociedad internacional debe descansar sobre bases consistentes; para tal efecto, se debe readecuar los términos de la relación Estado-sociedad y flexibilizar el principio de la soberanía absoluta.

Lo más característico de este nuevo diseño de Estado-nación es la gran importancia e influencia que han ganado los derechos humanos. El esquema del Estado liberal; que significa el reconocimiento de los derechos de ciudadanía ha triunfado, no se puede saber si coyuntural o permanentemente, pero sí se percibe la tendencia de que la legitimación. de los Estados nacionales más que nunca va a estar sometida a los criterios de legitimidad de origen y respeto de los derechos humanos.

El criterio de legitimación de la ciudadanía política y social es un elemento que en los últimos 40 años cobró una gran importancia, que seguirá aumentando. Es por eso que el positivismo jurídico que exacerbó el concepto de la soberanía está siendo relativizado por una nueva conciencia jurídica internacional fundada en el renacer del derecho natural. ${ }^{16}$ Este enfoque jurídico-político reconoce los derechos del individuo como anteriores a la constitución de cualquier sociedad política.

Esta racionalidad jurídica es más que un elemento legitimador del nuevo Estado nacional, y será un componente legitimador y limitador del Estado nacional. Esta racionalidad se origina en la nueva relación entre Estado y sociedad, y se estructura a partir del principio estricto de autolimitación del ejercicio del poder político. No se sostiene que esto pueda suceder en todas las sociedades en un plazo inmediato, pero sí se visualiza que es la dirección hacia donde avanza el Estado-nación.

Las nuevas necesidades de tipo funcionalista, que tienen que ver con el proceso económico, el desarrollo social y el imperativo político de consolidar una forma superior de organización politica, han significado la ruptura con los paradigmas anteriores del análisis de las relaciones internacionales. El enfoque realista, que tuvo tanta vigencia y que todavía la tiene, quedó en el análisis, no diremos perimido u obsoleto, pero sí como un enfoque que nos ofrece una visión insuficiente.

${ }^{16}$ Otfried Hoffe, Politische Gerechigkeit, (Frankfurt: 1989), p. 92. 
El paradigma realista se elaboraba partiendo de algunos supuestos que hoy en día noise dan más en el mundo de las relaciones exteriores. En primer lugar; el análisiș se centraba en el Estado; en segundo lugar, se separaba la política interna de la política exterior; en tercer lugar, partía de la base de que la política internacional era solamente lucha por el poder, y su consecuente entorno internacional anárquico; y en cuarto lugar, partía de un supuesto, que si bien podía ser un postulado jurídico nunca fue una realidad, la igualdad y la legalidad soberana de todos los Estados. Estos cuatro supuestos del enfoque realista de la política, que concebía a un Estado-nación como único actor de las relaciones exteriores, ha sido superado. El solipsismo jurídico ha sido inadyertidamente dejado de lado por los nuevos problemas del escenario internacional. Los problemas del narcotráfico, del medio ambiente y de la extrema pobreza son capítulos que están influyendo en las agendas de las políticas exteriores.

Hoy en día, el esquema realista que solamente centraba la política exterior en el Estado-nación y en la necesidad de maximizar sus intereses, ${ }^{17}$ ha sido superado por las circunstancias generadas por la dimensión planetaria de la modernidad. Así, el realismo desconoce la necesidad de la interdependencia que existe entre las sociedades nacionales. En el. presente, las sociedades nacionales son interdependientes y sus relaciones se fundan en razones de complementariedad.

En la actualidad no se puede planificar una política exterior extremadamente competitiya, pues el mundo se ha convertido en un sistema en el que las demandas internas de las sociedades tienen que ser satisfechas por los Estados. ${ }^{18}$ La necesidad de satisfacer las demandas internas está afectando la forma y los hábitos relativos a cómo solucionar los problemas exteriores. La interdependencia no es percibida por la visión realista de las relaciones internacionales.

El realismo percibió al Estado como actor dominante 19 y concibió a la necesidad de acumular poder y a la de aumentar el prestigio como objetivos prioritarios de la política exterior. A partir de la priorización de estas necesidades se diseñó y dedujo la convivencia internacional construida sobre las bases del sacrosanto principio de la soberanía absoluta.

\footnotetext{
${ }^{17}$ Paul Kennedy, The Rise and Fall of the Great Powers, op. cil., p. 73.

${ }^{18}$ Carlos Mateo Balmelli, op. cit., pp. 237-279.

${ }^{19}$ Robert Keohane / Joseph Nye, op. cit., p! 39.
} 


\section{5:- Los derechos humanos y el desarrollo institucional.}

En este capítulo la intención es anticipar algunas características y elementos constitutivos del Estado-nación en las postrimerías de este siglo. El proceso de globalización vulnera y sensibiliza a las sociedades nacionales, los países interactúan entre si y las fronteras se vuelven permeables por el fenómeno de los medios de comunicación.

La transnacionalización de los procesos económicós y sociales rèquiere, aunque parezca păradójico, de una mayòr gestión local. Esto último refiere la importancia de una estrategia de desarrollo institucional, en consecuencia se debe instituciónalizar y racionalizar los procesos de diseño, toma y ejecución de la decisión. El poder político debe ser organizado nacional y localmente para solucionar los problemas emergentes de la lucha por el poder y para dotar a la sociedad de formas más eficientes de organización que permitan la elaboración de las demanindas sociales. Se requiere de un nuevo estilo de gerenciamiento. 20

El concepto rígido de soberanía está derimido. La conciencia jurídica común es un elemento importante a considerar en la configuración de la sociedad internacional, de lo contrario este proceso será frágil, sobre todo si solamente es deseada y fundada a partir de argumentos o necesidades económicas que tienen que ver con el desarrollo del mercado internacional.

El fenómeno que se denominó el restablecimiento del derecho natural" supone reconocer la dimensión ética de la convivencia social y del individuo. Esto ayuda de una manera determinante a lograr una visión sistémica del mundo y no atomizada, formada a partir de unidades nacionales. Las cancillerías respectivas de todos los países tendrán que ir decidiendo a partir del procesamiento de información y de una transacción de valores, proceso en el cual no solamente se van a tener en cuenta las necesidades provenientes de las diplomacias para el desarrollo, sino también se va a considerar la ética en dos dimensiones.

El sociólogo alemán Max Weber categorizó dos tipos de éticas: la de la convicción y la de la responsabilidad. Lá primera se deduce de un esquema valorativo y cuenta con una validez categórica, mientras que la ética de la responsabilidad es la consecuencia de un sistema valorativo y de la probabibilidad práctica de poder materializar los valores.

${ }^{20}$ Bernardo Kliksberg, Una gerencia pública para los nuevos tiempos, en: Reforma de las instituciones del gobierno, editado por la Comisión Presidencial para la Reforma del Estado (COPRE), (Caracas: 1994), pp. 204-213. 
En el presente, estos dos tipos de ética deberán ser tenidos en cuenta por los ejecutores de la política exterior. ${ }^{21}$ Por un lado, en la actualidad el respeto a los derechos humanos es considerado como un valor con validez universal. Es decir, su respeto y promoción no están sometidos a ningún tipo de relativismo situacional, histórico o contextual. Por el otro lado, al existir una sobreabundancia de posibilidades y necesidades, el manejo de la política exterior tendrá que inspirarse en criterios pragmáticos que respondan a la vieja razón de Estado. Hoy en día la sociedad internacional entraña tal complejidad que obliga a elaborar la agenda exterior a partir del reconocimiento de los intereses concretos y de la promoción de principios generales.

Es innegable que el reconocimiento universal de los derechos humanos flexibiliza el principio de soberanía. La problemática de los derechos humanos y su promoción se ha convertido en el elemento volitivo de conciencia de los Estados, pero no por ello debemos menoscabar los intereses concretos de los gobiernos.

La sociedad internacional y las relaciones internacionales se han complejizado. Es por ello que los ejecutores de las relaciones internacionales deberán actuar en una forma ambigua, inspirados tanto en una valoración ética del individuo como respondiendo a las necesidades prácticas del poder y el bienestar material.

La mayor densidad y complejidad de las relaciones internacionales exigen estructuras estatales más sofisticadas. El desarrollo institucional de las sociedades es una condición para aumentar la capacidad endógena de los países. ${ }^{22}$ En un mundo globalizado un bajo nivel de desarrollo institucional es una mala carta de presentación. Las sociedades nacionales deben empeñarse en institucionalizar el proceso político y social, de lo contrario la ausencia de tales instituciones supone la incorporación asimétrica al proceso de transnacionalización que vive el mundo.

La incorporación asimétrica de los países a los procesos de globalización estará en relación proporcional al grado de evolución institucional. Los Estados deben capacitarse y agotar las posibilidades de los recursos propios, y para este efecto el fortalecimiento y racionalización de los mecanismos de ejecución y decisión es de fundamental importancia.

\footnotetext{
${ }^{21}$ James Dougherty/Robert Pfaltzgraff, Teorias en pugna en las relaciones internacionales, (New York: 1990), p. 102.

22 Joan Prats Cataià, Las reglas del juego: estrategias del desarrollo institucional en América Latina" en: Seminario organizado por la PNUD sobre El desarrollo humano sostenible: el contenido de las políticas públicas de nueva generación", Buenos Aires, 1995.
} 
Las economías locales deben estar preparadas para la ampliación de los mercados. Está demostrado que una de las razones del subdesarrollo es la estrechez de los mercados. Es por ello que hay que ser concientes de las relaciones de interdependencia y que estas nuevas circunstancias están retando a la concepción clásica del Estado-nación.

La reformulación del Estado-nación tiene como efecto inmediato nuevos organismos y nuevas formas de interactuar en la comunidad internacional. La finalidad del cambio institucional en el marco global es la de ampliar y garantizar la vigencia de los derechos de ciudadanía.

La construcción de la nueva sociedad internacional no puede ser deseada solamente desde un prisma economicista. De darse esto la consecuencia inmediata es, y seguirá siendo, el establecimiento de múltiples y variadas situaciones de injusticia en el relacionamiento entre los Estados. El nuevo status jurídico de los Estados y de la comunidad internacional tiene como finalidad el respeto y la promoción de los derechos humanos.

Constantemente se ha referido en este ensayo la necesidad de que las sociedades nacionales se preparen internamente para enfrentar los desafios que se imponen con el proceso de globalización. En este ensayo, la globalización es conceptualizada como un proceso social que afecta aspectos políticos, culturales y económicos de la convivencia humana local e internacional. El modus vivendi de la humanidad se encuentra sometida a problemas, demandas, expectativas de consumo, visiones ideológicas que cobran una dimensión geográfica global y exigen soluciones planetarias.

Desde este punto de vista, el proceso de globalización puede ser entendido como la vertiente exógena del proceso de modernización. La globalización de los efectos de los fenómenos locales e internacionales expresa que la modernidad ha cobrado gravitación y dimensión planetaria. Cuando se habla de la globalización se refiere el impacto de lo externo en los asuntos domésticos. De este último párrafo no se puede inferir que los procesos sociales están inducidos hacia una suerte de linealidad evolutiva, ni que la globalización es un imperativo ético-normativo al cual se deben adecuar los comportamientos nacionales y locales, o que el proceso de globalización sea una imposición externa para forzar el progreso o provocar el atraso social.

Como se dijo, la globalización es la demostración de que los efectos de la modernidad cobran presencia y trascienden a escala mundial. El efecto de la transnacionalización influye en forma dispar en las sociedades nacionales. 
La globalización tendrá efectos perturbadores en las unidades sociales que no estén embarcadas en procesos endógenos de modernización y provocará efectos contrarios si la globalización como proceso externo no es concomitante con el proceso interno de ordenación moderna. Se deben establecer relaciones de correspondencia entre la vertiente exógena y endógena de la modernidad; para tal fin es que se sugiere direccionar las reformas políticas, sociales y económicas hacia el aumento de la capacidad endógena para que la inserción de las sociedades nacionales al proceso dé globalización se produzca en términos simétricos.

La incorporación al mundo global abarca mucho más que la mejoría de la participación económica local en el mercado internacional. Incorporarse a este estadio de la internacionalización significa subordinar la estructura jurídica e institucional del-Estado-nación a las normas que amplían y reconocen los derechos de ciudadanía. La visión economicista de la globalización no permite atender y analizar la pluralidad de efectos que resultan de este proceso estimulado por una heterogeneidad de elementos. Por ello, resulta inconveniente proceder a planificar políticas de desarrollo que tengan como fundamento el hermetismo político, jurídico y económico del Estado-nación.

Las unidades nacionales no pueden ejecutar políticas de contracción relativa o ensimismamiento absoluto, lo que se sugiere es que las políticas públicas de reformas se direccionen a impulsar un proceso de ordenamiento y racionalización social:que viabilice la posibilidad de asociación de las unidades nacionales a la sociedad internacional.

La disociación de los países supondrá àivivel nacional la dualización social, el atraso económico y la barbarrie en la política. Estas consideraciones conducen a pensar que la proyečción de un modelo de desarrollo autocentrado puede ser factible, pero no serảilo más adecuado para asociar y armonizar los procesos de modernización endógenos y exógenos, y que además deben ser concomitantes.

\section{6.- Criterios para la formulación de políticas públicas para la inserción.}

En el transcurso de este ensayo se ha manifestado en forma recurrente la necesidad de que los países de la región aumenten su capacidad endógena para poder insertarse en forma exitosa en el proceso de globalización. Las políticas de inserción requieren ser diseñadas y pensadas en términos 
históricos, vale decir, elaborar una estrategia que pondere la capacidad nacional existente y las restricciones provenientes de la dinámica de la globalización.

Siguiendo a Luciano Tomassini, ${ }^{23}$ se deben emprender las políticas públicas que favorezcan la construcción de la capacidad interna; de esta manera se posibilitará que los gobiernos nacionales sean capaces de manejar por sí mismos la inserción de los países de la 'región en los procesos de globalización.

El mejoramiento de la capacidad endógena debe tener como primer objetivo la ejecución de políticas que se orienten a reformar el Estado.

En lo político, el Estado debe ser fortalecido entendiendo por esto la vigorización de las instituciones como sinónimo de la consolidación y la profundización de la democracia.

En lo administrativo, el Estado debe ser reforzado teniendo como objetivo ampliar su capacidad de gestión. Hay que emprender políticas que subordinen el manejo de la administración pública a los principios de la legalidad para de esa manera reducir el patrimonialismo de Estado; por último se debe tender a introducir mayor flexibilidad administrativa, hay que establecer un gerenciamiento público con capacidad para reaccionar adecuadamente a las nuevas demandas de la problemática social.

En lo social, el Estado debe afirmar su presencia. El objetivo debe orientarse a complementar la ciudadanía política con elementos de ciudadanía social. Primeramente se sugiere priorizar la dimensión normativa institucional de la democracia, esto es la rutinización del ejercicio del poder político, la trasparencia de los procesos electorales y todo lo que abarque el proceso de formalización de las relaciones civiles; sociales y políticas.

Luego se sugiere complementar la noción formal e institucional de la democracia con criterios económicos y sociales que si bien no son definidoras de la democracia, son, sin embargo, elementos necesarios para lograr la integración social y la estabilidad política del sistema democrático.

En lo económico, el Estado deberá retraerse del proceso èconómico y dejar de ser el impulsor de las políticas públicas que estimulen el crecimiento económico.

\footnotetext{
${ }^{23}$ Luciano Tomassini, Una visión post-modernista de las relaciones internacionales", en: Integración solidaria: Reconstrucción de los sistemas politicos latinoamericanos, (Caracas: 1993), p. 36.
} 
Las actuales circunstancias exigen redefinir un Estado con funciones subsidiarias, residuales y orientadoras del proceso económico. El esfuerzo de la capacidad estatal debe volcarse a estimular el fortalecimiento del sector privado y a echar las bases para ampliar las posibilidades de la economía de mercado.

Es obvio, que para tales objetivos se deben considerar en el diseño de las políticas públicas no sólo los fines, sino también los medios; y en especial, se debe tener muy presente que la estabilidad macroeconómica es un requisito para cualquier política de desarrollo que se haya impuesto metas sociales.

La tarea de la inserción en forma exitosa está sometida a varios requisitos. La primera de esas exigencias es la de entender la simultaneidad cronológica de diseñar y ejecutar políticas públicas internas y políticas públicas externas para enfrentar con éxito la globalización. Así por ejemplo, las políticas exteriores de la democracia tuvieron como presupuesto el final del autoritarismo. Desde el inicio de las transiciones, la política exterior de los países de la región estuvo orientada a consolidar la democracia. El objetivo estaba puesto en recobrar la confianza de la sociedad internacional para que los países de la región sean vistos como actores dignos y confiados. ${ }^{24}$

La complejidad de la sociedad internacional exige que la planificación de las políticas exteriores ponderen diferentes variables para la elaboración de la nueva agenda internacional. Se debe establecer una sincronía entre la realización de la nueva agenda internacional y el mejoramiento de la capacidad instalada de las cancillerías nacionales.

La política exterior para la inserción debe tener como fundamento los mecanismos de la diplomacia. Se pretende que a través de los acuerdos existentes se ajusten las diferencias entre los Estados. El realismo político debe ser una constante en el comportamiento exterior de los Estados. El mismo abarca dos aspectos que deben ser tenidos en cuenta:

a. Todo ordenamiento supranacional se funda en la estructura del Estado-nación; $y$

b. la integración regional o global de los Estados debe perseguir el objetivo de maximizar los intereses de los países.

La política exterior debe promover el concepto de la seguridad jurídica, que debe ser considerada en dos aspectos. La seguridad jurídica supone, a nivel interno, el Estado democrático de derecho; esto último comprende un desarrollo institucional que abarque el establecimiento

\footnotetext{
${ }^{24}$ José Luis Simón, La politica internacional para la democracia, (Asunción: 1995), pp. 47-108.
} 
tripartito de los poderes del Estado en relación de igualdad jurídica e interdependencia, derechos y garantías constitucionales y la certeza de contar con organismos jurisdiccionales independientes. En un mundo globalizado, la ausencia de instituciones es un impedimento para el progreso social.

En el orden externo, la seguridad jurídica supone que los acuerdos pactados en el área internacional deben ser cumplidos. Asimismo, la tendencia del ordenamiento jurídico-regional o internacional debe orientarse a armonizar los esquemas de derecho nacional con los internacionales. Se debe propender a que ciertas normas regionales o internacionales vayan cobrando a nivel nacional validez jurídica plena.

La diplomacia, en el futuro, no debe ser únicamente un mecanismo al servicio de la estrategia de poder entre los Estados. Se debe pensar en la diplomacia para el desarrollo, como instrumento de política pública que permita dotar a la economía nacional de capacidad competitiva, y que tenga en cuenta que los países con ventajas comparativas son aquellos que incorporan a sus procesos productivos tecnologías y disponen de capital humano para implementar las políticas de crecimiento económico.

La diplomacia para el desarrollo debe ser vista como un recurso que estimule la capacidad competitiva de las economías aprovechando las ventajas comparativas, la apropiación de tecnología y la consolidạción de recursos humanos para el progreso. El nuevo diseño de las políticas exteriores requiere atender las razones sociales y económicas de los conflictos. Este enfoque permitirá enfrentar desde otra perspectiva los problemas internos de seguridad y las discrepancias exteriores que surjan entre los Estados.

Al indagar el origen social y económico de los problemas se propone la idea de establecer sistemas económicos eficientes a los cuales se les puede atribuir metas sociales; esto es posible, siempre y cuando los gobiernos y las circunstancias históricas permitan garantizar la gobernabilidad. La gobernabilidad interior y exterior es una condición para establecer una dominación política que ordene la sociedad y satisfaga las demandas sociales.

La gobernabilidad sugiere un proceso de creación institucional permanente entre el Estado y la sociedad, y obliga también a establecer un vínculo entre estilo de gobierno y potencialidad de desarrollo. Al garantizar la gobernabilidad se busca dotar de contenido ético y programático a la dominación política. 
Los países de la región deben mejorar su capacidad instalada para actuar en un sistema internacional en el que las relaciones bilaterales seguirán siendo un elèmento motorizador de la conducta exterior de los Estados, pero en el contexto actual los Estados nacionales deben estar. infraestructuralmente preparados para actuar a nivel regional y a nivel global. Los gobiernos deben planificar la política exterior pensada para escenarios globales y practicar una democracia intermediada que reconozca la existencia de diferentes niveles de relacionamiento exterior. ${ }^{25}$

La reforma administrativa de la que se habló debe dotar al Estadonación de los recursos suficientes para poder relacionarse a nivel interestatal, regional y global. Cuando se piensa en la creación de bloques regionales, $\mathrm{y}$ en el proceso de creación de instituciones supranacionales, también se tiene que reconocer los límites funcionales de los Estados.

El objetivo de la integración debe ser tomado en serio por las cancillerías locales. El proceso de globalización obliga a los países a partir de criterios regionales, a formar bloques para fortalecer la presencia internacional de la región y para mitigar los efectos perturbadores de la globalización.

Los procesos de integración deben realizarse con su alta dosis de pragmatismo. Los proyectos de integración no deben proponerse objetivos que cumplan una función prescriptiva de la voluntad de los Estados. Los objetivos que cumplen una función prescriptiva de las voluntades rigidizan los procesos al restarles flexibilidad e iniciativa a la capacidad de los Estados. La integración con su dinámica debe orientarse a objetivos inmediatos y mediatos, proceso que debe someterse a una metodología de consultas y evaluación de resultados permanentemente.

Comparativamente hablando la ventaja de los procesos de integración en la región es que los mismos en comparación con Europa no persiguen el mantenimiento de la paz. Los proyectos de integración no deben estar alimentados por la realización de grandes sueños.

La integración debe favorecer la complementariedad entre los paises de la región. Se debe tender a la convergencia macroeconómica y á la armonización normativa para disminuir la capacidad discrecional de los Estados que conduzcan a la formación de instancias supranacionales. La integración debe perseguir el objetivo de la complementariedad

\footnotetext{
${ }^{25}$ Lincoln J. Bizzozero, Las relaciones entre los paises del Atlántico suboccidental y Europa Central", Cuadernos del CLAEH, No5S, Montevideo, 1990, p. 80.
} 
económica y social, de la consolidación de la democracia y debe afirmar la presencia de los países de la región en los asuntos internacionales.

Según Henry Kissinger ${ }^{26}$ por lo menos seis países compartirán los roles protagónicos en el próximo siglo (Europa, Japón, Rusia, China, Estados Unidos y probablemente la India). Los países latinoamericanos no pueden pretender en lo inmediato una presencia político-militar de trascendencia, pero sí podrán aunando esfuerzos y con un sistema de conductas coherente tener una voz en los asuntos internacionales. Si bien es cierto que otras regiones del mundo han crecido con un ímpetu sin igual, e Hispanoamérica sigue siendo afectada por las consecuencias de las décadas perdidas, se puede afirmar, siguiendo al ex-Presidente Osvaldo Hurtado, que la década fue perdida en lo económico pero ganado en lo político. 27

Se coincide con Luciano Tomassini ${ }^{28}$ cuando sostiene que el desafío que enfrentan los países latinoamericanos es el de incorporarse al mundo y al futuro. De lo contrario: En el contexto actual no hacerlo, equivaldría a perecer".

\section{7.- Conclusiones.}

Es imprescindible que toda reforma del Estado vaya antecedida y acompañada de un proceso de reflexión y debate teórico. El debate de las cuestiones político-institucionales cumple una función orientadora que permite esclarecer los conceptos y hacer un inventario de los recursos existentes.

La reflexión previa a la acción política, en especial en un medio social de alto grado de complejidad, es una exigencia resultante de la necesidad de apuntalar el proceso de reforma y que además sirve para ponderar y visualizar las posibilidades de cambio, que son más posibles de lo que se piensa.

La discusión y la teorización no persigue ni ideologizar ni inhibir la voluntad política de los actores; al contrario, el ejercicio del pensamiento libre aleja la nefasta metamorfosis que pretende transformar la afirmación en verdad incuestionable.

La reflexión, en política, sugiere que el proceso político de reforma institucional que desvincula la organización del poder de la racionalidad

${ }^{26}$ Henry Kissinger, Diplomacy, (New York: 1994), p. 23.

${ }^{27}$ Osvaldo Hurtado Larrea, Gobernabilidad y Reforma Constitucional, (Quito: 1993), p. 443.

${ }^{28}$ Luciano Tomassini, Una visiỏn post-modemista de las relaciones internacionales", op. cit., p. 40. 
y el ejercicio del poder del sentido común; está despertando en el interior de la sociedad la fuèrźa destructiva de la irracionalidad.

En este ensayo se ha preteridido demòstrar que la mayor complejidad de làs relaciones exteriores demanda una sociedad política más sofisticada. Las exigencias de dentro y el imperativo contextual externo agotan la cápácidad operativa y de infraestructura del Estado-nación, concebido desde el sacrosanto principio de la soberanía absoluta.

Por segunda vez en la historia se debe desacralizar al Estado-nación moderno. La primera secularización del Estado consistió en hacer reposar la legitimidad de la práctica estatal en la voluntad política de los miembrós de la sociedad. Se abandonaron los elementos de legitimidad dedúcídos de principios metafísicos que trascendían e imponían las estructuras estatales a la voluntad política de la sociedad. La segunda secularización consiste en desmitificar el sacro principio de la soberanía absoluta.

En el transcurso de este trabajo se ha sostenido la necesidad de remover y cambiar las bases en las que descansa el Estado. Para ello, debe darse inevitablemente una reforma institucional en la que el primer imperativo es la elaboración de una base constitucional adecuada.

Sin la reforma constitucional necesaria se hace imposible que los Estados puedan enajenar soberaníu y transferir capacidad decisional. Se deben lograr arreglos institucionales que zonifiquen áreas de cooperación y coordinen las políticas públicas.

La adecuación constitucional es un imperativo del proceso de reforma institucional que tiende a las concreción de sistemas legales armonizados y complementarios. La idea de reforma sugiere que el proceso de creación institucional no se convierta en un proceso en el que las instituciones se vayan agregando, produciendo una superposición de funciones.

El proceso de creación institucional debe someterse a una racionalidad que induzca a una diferenciación funcional de las instituciones, en la que las instancias del Estado y la de los organismos supranacionales se coordinen y complementen. Las restricciones que imponen el mayor grado de complejidad social obliga a considerar a la variable institucional como el recurso más idóneo del proceso político, capaz de elaborar y satisfacer las nuevas demandas.

Lo paradójico en el proceso de ordenamiento institucional, tanto en lo que afecta a la sociedad política nacional como a los organismos 
supranacionales, es que el Estado sigue siendo el agente central y el objeto del cambio estructural.

La nueva agenda de las relaciones internacionales debe ser elaborada a partir del reconocimiento del mayor grado de complejidad. Parte de esta tarea consiste en entender que el entorno internacional y la sociedad nacional se encuentran sometidos a una relación de'sobreabundancia de posibilidades y de conexiones, de modo que ya no es posible plantear una correspondencia biunívoca y lineal entre un elemento y otro.

El comportamiento exterior de los Estados y sus recursos institucionales responderán a circunstancias muy variadas. Desde la vieja razón de Estado que sugiere la búsqueda de alianzas político-militares, pasanido por una concepción colectiva de la seguridad; hasta las nuevas problemáticas del medio ambiente y la integración de los mercados, todos serán puntos clave a ser tenidos en cuenta en la elaboración de la agenda de la política exterior de los Estados.

Es debido a estas circunstancias que los cambios deben llevarse a cabo considerando los siguientes factores:

a. La reformulación del Estado-nación debe tomar como objetivo armonizar el ordenamiento jurídico-nacional con el ordenamiento jurídico-regional e internacional. La construcción del orden mundial, para contar con cimientos sólidos, debe ser estructurado a partir del Estado-nación. El Estado-nación debe servir de base al andamiaje institucional que coordine el conjunto de instituciones y administre la complejidad existente, producto del aumento de la interacción entre los diferentes factores que dinamizan el proceso de globalización.

b. Las sociedades nacionales deben mejorar su capacidad endógena para insertarse simétricamente en el proceso de globalización. La globalización permea de tal manera a las sociedades nacionales, someten a los gobiernos a unas circunstancias que se caracterizan por el aumento de las demandas y expectativas sociales.

Esto implica la necesidad de perfeccionar los esquemas institucionales existentes y de aumentar la capacidad de gerenciamiento de las burocracias estatales. Las políticas públicas que pretendan combatir el subdesarrollo deben lograr que los gobiernos locales se conviertan en herramientas válidas para dinamizar el progreso social.

c. Los sistemas económicos deben:ordenarse sobre bases macroeconómicas estables. La estabilidad macroeconómica es un requisito para la inserción y para la integración. 
Para lograr la inserción se requiere de la estabilidad de ciertas variables que permitirán el ahorro doméstico y la posibilidad de financiar inversiones locales recurriendo en menor medida al ahorro externo. La excesiva dependencia del financiamiento externo vulnera las bases en la que se asienta un modelo de desarrollo sustentable que considere los requisitos económicos y las metas sociales de las políticas públicas.

Para la integración, la estabilidad macroeconómica es una condición para lograr la convergencia económica entre diferentes Estados que están involucrados en los procesos integracionistas.

d. Las políticas exteriores de los países de la región deben convertirse en un instrumento al servicio de objetivos políticos y socioeconómicos. El diseño de las políticas exteriores tuvo y tiene como objetivo la consolidación de la democracia.

Los países latinoamericanos se han incorporado a la corriente de Estados que anhelan fundar una nueva sociedad política internacional que reconozca en su estatuto jurídico la superioridad de los derechos de ciudadanía. Para los países de la región el respeto y la promoción de los derechos humanos será tema de debate en las relaciones internacionales.

Por otro lado, la política exterior de los países de la región debe estar al servicio de las necesidades del desarrollo; para tal efecto, se debe dotar a las cancillerías de los recursos infraestructurales e institucionales que permitan diseñar y ejecutar una diplomacia para el desarrollo.

La tarea se muestra difícil y las restricciones impuestas por la realidad convocan al pesimismo. Si las soluciones deben ser deducidas de paradigmas que subalternizan la política a procesos exteriores a ella, la respuesta a la problemática consistirá en adecuar la conducta de las sociedades al destino impuesto por las circunstancias históricas.

Pero partiendo de un enfoque que reconoce la autonomía de la política es posible imaginar en lo abstracto y comprobar en lo concreto que los procesos sociales son maniobrados y orientados por la voluntad de los actores políticos y sociales.

Desde los griegos se reconoce que la política es una actividad objeto de reflexión del pensamiento filosófico. La política es parte del dominio cognocitivo humano; por ello, se puede presumir que es susceptible de ser deliberadamente definida. De allí que es posible pensar en el logro de la sociedad desarrollada cuando un proceso político incorpora ética y racionalidad, como también es un acierto prever la decadencia política si es que el proceso político no esta conciliado con la racionalidad y la ética. 
Cuando el proceso político es conducido por la irracionalidad o por la ceguera de estar embarcado en la realización de sueños imposibles el resultado será el mantenimiento de formas atávicas de convivencia. 